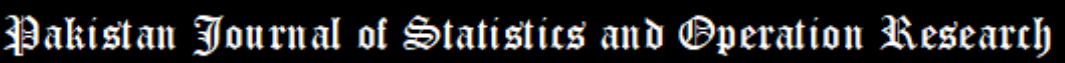

\section{Testing the proportionality assumption for specified covariate in the cox model}

\author{
Mohamed Ali HAFDI
}

High school of technology Laayoune \& IMI laboratory, Ibn Zohr university, Morocco, m.hafdi@uiz.ac.ma

Abstract

Many tests based on alternative models are considered to test the proportionality assumption for specified covariate. The common point of all these alternatives is the linear form of an added component according to the covariate concerned by the test while the proportionality can be also violated by a non linear form of this added component. In this paper, we propose a test for proportional hazards assumption for specified covariates. The test is based on a new alternative which can be applied in the two situations cited above and also when the hazards rates under different values of covariates is not only constant as in the Cox model, but it may cross, go away, and may be monotonic with time. The limit distribution of the test statistic is derived. Finite samples properties of the test power are analyzed by simulation and compared with those of existing tests. Application on Real data examples are considered.
\end{abstract}

Key Words: Cox model; Partial likelihood; Proportionality assumption; Anderson-Darling test.

Mathematical Subject Classification: 62N01, 62N02, 62N03, 62E20.

\section{Introduction}

The most known semi-parametric model for analysis of failure time regression data is the Cox model introduced by Cox (1972). A key assumption in this model is the proportionality of the effects of the covariates on the hazard rates. Let $S_{x}(t)$ and $\lambda_{x}(t)$ be the survival and hazard rate functions under a $p$-dimensional covariate $x=\left(x_{1}, \ldots, x_{p}\right)$. Denote by $\Lambda_{x}(t)=\int_{0}^{t} \lambda_{x}(u) d u=-\log \left(S_{x}(t)\right), t \geq 0$, the cumulative hazard rate under $x$. The Cox model express the hazard rate according to $x$ as follow

$$
\lambda_{x}(t)=e^{\beta^{T} x} \lambda_{0}(t)
$$

where $\beta$ is a vector of unknown parameters and $\lambda_{0}(t)$ stands for an unknown baseline hazard function. Under the model (1) the cumulative hazard rate under $x$ has the forme

$$
\Lambda_{x}(t)=e^{\beta^{T} x} \Lambda_{0}(t)
$$

where $\Lambda_{0}(t)=\int_{0}^{t} \lambda_{0}(t) d t$ is the cumulative baseline hazard function.

One of the main assumptions in the model (1) is of course the proportionality, that the ratio of two hazard under a covariates $x$ and $y$ is constant in time,

$$
\frac{\lambda_{x}(t)}{\lambda_{y}(t)}=e^{\beta^{T}(x-y)} .
$$

Note that a model misspecification give an inappropriate interpretation of obtained results. This allowed several global hazard proportionality tests to be developed. Among these tests we can cite those given by Cox (1972), Moreau et al. 
(1985), Lin (1991), Nagelkerke et al. (1984), Grambsch and Therneau (1994), Quantin et al. (1996), Bagdonavičius et al. (2004).

If the proportionality hypothesis is rejected, so the identification of the covariates causing a such assupmtion violation will be a matter of great interest. Statistical tests for checking the proportionality of a single covariate have been considered by a number of authors, see for instance Kvaløy and Neef (2004), Kraus (2007), Grambsch and Therneau (1994), Bagdonavičius and Levuliene (2019).

One of the most important inference tools is the following $p$ score functions:

$$
U_{j}(\beta, t)=\sum_{i=1}^{n} \int_{0}^{t}\left(x_{j}^{(i)}-\frac{\sum_{k=1}^{n} Y_{k}(u) x_{j}^{(k)} e^{\beta^{T} x^{(k)}}}{\sum_{k=1}^{n} Y_{k}(u) e^{\beta^{T} x^{(k)}}}\right) d N_{i}(u), j=1, \ldots, p,
$$

where $n$ is the number of subjects and $x^{(i)}, N_{i}(t)$, and $Y_{i}(t)$ are respectively the covariate, the failure indicator, and the risk indicator at the moment $t$ of the $i^{t h}$ subject.

The estimation of the unknown vector parameters $\beta$ in model (1) $\operatorname{Cox}$ (1972) proposed to solve the system $U(\beta, \infty)=$ $\left(U_{1}(\beta, \infty), \ldots, U_{p}(\beta, \infty)\right)=(0, \ldots, 0)$. Let $\hat{\beta}$ be the obtained estimate of $\beta$.

Denote by $I(\beta, t)$ minus the derivative of $U(\beta, t)$ with respect to $\beta$. Note that $J(\beta)=I(\beta, \infty)$ is the information matrix.

To examine the proportionality assumption of $x_{j}$ the $j^{\text {th }}$ component of the covariate $x$, Lin et al. (1993) propose to use the tests of Kolmogorov-Smirnov type $(K S)$ based on the statistic

$$
K S=\sqrt{J_{j j}(\hat{\beta})} \sup _{t}\left|U_{j}(\hat{\beta}, t)\right|,
$$

where $J_{j j}(\hat{\beta})$ is the $j^{t h}$ diagonal element of the information matrix. On a $5 \%$ level, the null hypothesis is rejected when $K S \geq 1.36$.

There are also the test of Anderson-Darling $(A D)$ and Cramer-von Mises $(C V)$ type based respectively on the following statistics:

$$
C V=J_{j j}(\hat{\beta}) \int_{0}^{\infty} U_{j}(\hat{\beta}, t)^{2} d \hat{q}_{j}(t), \quad A D=J_{j j}(\hat{\beta}) \int_{0}^{\infty} \frac{U_{j}(\hat{\beta}, t)^{2}}{\hat{q}_{j}(t)\left(1-\hat{q}_{j}(t)\right)} d \hat{q}_{j}(t)
$$

where $\hat{q}_{j}(t)=\frac{I_{j j}(\hat{\beta}, t)}{J_{j j}(\hat{\beta})}$. Note that these tests are studied by Kvaløy and Neef (2004) and the null hypothesis will be rejected on a $5 \%$ level if $C V \geq 0.461$, also if $A D \geq 2.492$.

The second set of tests cited below consists on a different alternative models given by inserting a time varying component depending on $x_{j}$ in the Cox model. We cited here three tests:

- The first that we note $(G)$, is proposed by Grambsch and Therneau (1994) using the following model:

$$
\lambda_{x}(t)=e^{(\beta+\gamma g(t))^{T} x} \lambda_{0}(t),
$$

and test $H_{0}: \gamma_{j}=0$ against $H_{1}: \gamma_{j} \neq 0$ for each $j=1, \ldots, p$ with wald test by estimating $\gamma$ using the scaled Schoenfeld residual and the generalized least squares. Some used form of $g$ function are $g(t)=t$ or $g(t)=\log (t)$. Another choice of $g(t)$ is the Kaplan-Meier estimate which is the default transform argument in the function cox. zph from the package survival in R.

- The idea of the second test proposed by Kraus (2007) that we note $(K R)$, consists of testing the proportionality assumption of the component $x_{j}$ using a $d$ smoothly time-varying coefficients. The test is score test and the used alternative model have the following form:

$$
\lambda_{x}(t)=e^{\beta^{T} x+\theta^{T} \psi(t) x_{j}} \lambda_{0}(t),
$$


where $\psi$ is the smooth functions vector such that $\psi(t)=\left(\psi_{1}(t), \ldots \psi_{d}(t)\right)$,

$$
\psi_{j}(t)=\varphi_{j}\left(\frac{F_{0}(t)}{F_{0}(\tau)}\right)
$$

$\varphi_{j}, j=1, \ldots, d$ are the smooth functions, and $F_{0}(t)=1-e^{-\Lambda_{0}(t)}$ is baseline distribution function. There are many possible choices of $\varphi_{j}$ (see, for instance, Peña (1998) and Kraus (2007)).

For deriving the reject or not of the proportionality assumption, we note that the test statistic is asymptotically $\chi_{d}^{2}$-distributed.

- The third test that we note $(B A G)$, is proposed by Bagdonavičius and Levuliene (2019). The authors consider the following model as an alternative of proportionality assumption:

$$
\lambda_{x}(t)=\frac{e^{\beta^{T} x+\Lambda_{0}(t) e^{\gamma x_{j}}}}{1+e^{\gamma x_{j}}\left(e^{\Lambda_{0}(t) e^{\gamma x_{j}}}-1\right)} \lambda_{0}(t) .
$$

This model by its complex expression form seems for the first time out of the class of the first two models for testing the proportionality assumption of $x_{j}$, but this is not true. Indeed, we can easily show that when $\gamma$ tends towards 0 (i.e we approach the null hypothesis), $\lambda_{x}(t)$ reduces to the following form:

$$
\lambda_{x}(t)=e^{\beta^{T} x-\gamma F_{0}(t) x_{j}} \lambda_{0}(t)
$$

An immediate consequence is that the two tests based on the two models (3) and (4) as alternatives, have the same power. Note that the statistic of this test is asymptotically $\chi_{1}^{2}$-distributed and the null hypothesis will be rejeted with a significance level $\alpha$ when the statistic value exceed the $(1-\alpha)$ critical value of the $\chi_{1}^{2}$ distribution.

In this paper, we present a test of the proportionality assumption in Cox model for each covariate separately. This test is based on the score function corresponding to a new alternative which will be described in the next section. In section 3, we present the test statistic. Investigation by simulation for the finite samples properties of the power of the proposed test compared to those of the other tests cited below, is given in section 4. An application to a three real examples is considered in section 5.

\section{A new test alternative model}

The formulation of the alternative model in $(G),(K R)$ and $(B A G)$ tests, is based on the following alternative:

$$
\lambda_{x}(t)=e^{\beta^{T} x+\Psi(t, \gamma) x}
$$

which the vector function $\Psi(t, \gamma)$ take a special form in each of those tests and $\Psi(t, 0)=0$.

Note that the added component for detecting the non proportionality in (5) is supposed to have a linear form according to $x$, but it will be important to take in mind that the violation of the proportionality can be caused not only by time dependent coefficients, but also by a non-linear form of the added component as for example if $\Psi(t, \gamma)$ depend also on $x$.

This idea is the principal motivation to generalizate (5) by the following classe of alternative models:

$$
\lambda_{x}(t)=e^{\beta^{T} x+\Phi(t, \gamma, x)} \lambda_{0}(t)
$$

where in $\Phi(t, 0, x)=0$.

The classe (6) can provide a more and interesting elements serving to deal with many new cases of violation of the proportionality assumption. In this article, we propose from this classe the following model defined as:

$$
\lambda_{x}(t)=e^{\beta^{T} x}\left(1+e^{\beta_{j} x_{j}} t\right)^{e^{-\gamma x_{j}}-1} \lambda_{0}(t)
$$

where $\beta_{j}$ and $x_{j}$ are respectively the $j$ th components of the parameter vector $\beta$ and the vector covariate $x$. Note that 
the model (7) can be generalized by remplacing $t$ by a general positive function $H(t)$ which can take many form (as $\left.H(t)=t, H(t)=\log (t), H(t)=\Lambda_{0}(t), \ldots\right)$.

The Cox model is a particular case of the model (7) when $\gamma=0$. The model (7) is very wide to the Cox model. Indeed, we consider two vector covariates $x$ and $y$. The ratio of hazard rates under model (7) is

$$
\frac{\lambda_{x}(t)}{\lambda_{y}(t)}=e^{\beta^{T}(x-y)} \frac{\left(1+e^{\beta_{j} x_{j}} t\right)^{e^{-\gamma x_{j}}-1}}{\left(1+e^{\beta_{j} y_{j}} t\right)^{e^{-\gamma y_{j}}-1}} .
$$

Note that $c_{0}=e^{\beta^{T}(x-y)}$, so the ratio of hazard rates has the following properties:

(i) If $\gamma x_{j}<\gamma y_{j}$ then the ratio of hazard increase with time of $c_{0}$ to $\infty$.

(ii) If $\gamma x_{j}>\gamma y_{j}$ then the ratio of hazard decrease with time of $c_{0}$ to 0 .

(iii) If $\gamma=0$ then we have the Cox model.

We remark that if $c_{0}<1$ in (i) or if $c_{0}>1$ in (ii) then the hazard rates (also the survival functions) intersect in a single point $t_{0}$.

\section{Test statistic construction}

Suppose that $n$ patients are observed. The $i$ th of them is observed under the covariate $x^{(i)}$. Denote by $T_{i}$ and $C_{i}$ the failure and censoring times for the $i$ th patient and set

$$
\begin{gathered}
X_{i}=\min \left(T_{i}, C_{i}\right), \quad \delta_{i}=\mathbf{1}_{\left\{T_{i} \leq C_{i}\right\}}, \\
N_{i}(t)=\mathbf{1}_{\left\{T_{i} \leq t, \delta_{i}=1\right\}}, \quad Y_{i}(t)=\mathbf{1}_{\left\{X_{i} \geq t\right\}},
\end{gathered}
$$

where $\mathbf{1}_{A}$ denotes the indicator of the event $A$. Then $N(t)=\sum_{i=1}^{n} N_{i}(t)$ and $Y(t)=\sum_{i=1}^{n} Y_{i}(t)$ are the numbers of observed failures in the interval $[0, t]$ and patients at risk just before the moment $t$, respectively. We suppose that failure times $T_{i}$ are absolutely continuous random variables.

Our purpose here is testing the proportionality assumption of a specified covariate let be $x_{j}$ the $j$ th component of the vector covariate $x$. Using the model (2), the null hypothesis will be so $H_{0}: \gamma=0$ tested by the score test.

The partial likelihood function (PL) (see Andersen et al. (1993)) adapted to model (2) is

$$
L(\beta, \gamma)=\prod_{i=1}^{n}\left(\int_{0}^{\infty} \frac{g\left(x^{(i)}, \beta, \gamma, u\right)}{\sum_{j=1}^{n} Y_{j}(u) g\left(x^{(j)}, \beta, \gamma, u\right)} d N_{i}(u)\right)^{\delta_{i}}
$$

where $g(x, \beta, \gamma, u)=e^{\beta^{T} x}\left(1+e^{\beta_{j} x_{j}} u\right)^{e^{-\gamma x_{j}}-1}$.

So the $\gamma$-component score function derived from (4) is

$$
\begin{aligned}
U_{\gamma}(\beta, \gamma) & =\frac{\partial}{\partial \gamma} \log (L(\beta, \gamma)) \\
& =\sum_{i=1}^{n} \int_{0}^{\infty}\left(\frac{\partial}{\partial \gamma} \log \left(g\left(x^{(i)}, \beta, \gamma, u\right)\right)-\frac{\sum_{k=1}^{n} Y_{k}(u) \frac{\partial}{\partial \gamma} g\left(x^{(k)}, \beta, \gamma, u\right)}{\sum_{k=1}^{n} Y_{k}(u) g\left(x^{(k)}, \beta, \gamma, u\right)}\right) d N_{i}(u),
\end{aligned}
$$

Under the null hypotheses $H_{0}: \gamma=0$, this partial score function depend on the unknown $\beta$ value which will be replaced by its partial maximum likelihood estimator $\hat{\beta}$ (see Cox (1972)). So the test statistic is

$$
\begin{aligned}
\hat{U}_{j} & =U_{\gamma}(\hat{\beta}, 0) \\
& =\sum_{i=1}^{n} \int_{0}^{\infty}\left(\hat{w}^{(i)}(u)-\tilde{E}(u, \hat{\beta})\right) d N_{i}(u),
\end{aligned}
$$


where

$$
\begin{gathered}
\hat{w}^{(i)}(u)=-x_{j}^{(i)} \log \left(1+e^{\hat{\beta}_{j} x_{j}^{(i)}} \Lambda_{0}(u)\right), \tilde{E}(t, \beta)=\frac{\tilde{S}^{(1)}(t, \beta)}{S^{(0)}(t, \beta)} \\
S^{(0)}(t, \beta)=\sum_{i=1}^{n} Y_{i}(t) e^{\beta^{T} x^{(i)}}, \tilde{S}^{(1)}(t, \beta)=\sum_{i=1}^{n} Y_{i}(t) \hat{w}^{(i)}(t) e^{\beta^{T} x^{(i)}}
\end{gathered}
$$

To finalize the test construction, we need the asymptotic distribution of $\hat{U}_{j}$ under the Cox model.

Set

$$
\begin{gathered}
E(t, \beta)=\frac{S^{(1)}(t, \beta)}{S^{(0)}(t, \beta)}, \quad S^{(1)}(t, \beta)=\sum_{i=1}^{n} x^{(i)}(t) Y_{i}(t) e^{\beta^{T} x^{(i)}}, \\
S^{(2)}(t, \beta)=\sum_{i=1}^{n}\left(x^{(i)}(t)\right)^{\otimes 2} Y_{i}(t) e^{\beta^{T} x^{(i)}}, \\
\tilde{S}^{(2)}(t, \beta)=\sum_{i=1}^{n} \hat{w}^{(i)}(t)\left(x^{(i)}(t)\right)^{T} Y_{i}(t) e^{\beta^{T} x^{(i)}}, \\
\tilde{\tilde{S}}^{(2)}(t, \beta)=\sum_{i=1}^{n}\left(\hat{w}^{(i)}(t)\right)^{\otimes 2} Y_{i}(t) e^{\beta^{T} x^{(i)}}
\end{gathered}
$$

where $A^{\otimes 2}=A A^{T}$ for any vector $A$.

Denote by $\beta_{0}$ the true value of $\beta$. Under the usual regularity conditions, the Doob-Meier decomposition, the delta method, and the following representation:

$$
n^{1 / 2}\left(\hat{\beta}-\beta_{0}\right)=\left(\Sigma\left(\beta_{0}\right)\right)^{-1} \sum_{i=1}^{n} \int_{0}^{\tau}\left\{x^{(i)}(u)-E\left(u, \beta_{0}\right)\right\} d M_{i}(u)+o_{p}(1)
$$

imply that

$$
\begin{gathered}
n^{-1 / 2} \hat{U}_{j}=n^{-1 / 2} \sum_{i=1}^{n} \int_{0}^{\infty}\left\{\hat{w}^{(i)}(u)-\tilde{E}(u, \hat{\beta})\right\} d N_{i}(u)= \\
n^{-1 / 2} \sum_{i=1}^{n} \int_{0}^{\infty}\left\{\hat{w}^{(i)}(u)-\tilde{E}(u, \hat{\beta})\right\} d M_{i}(u)+ \\
n^{-1 / 2} \int_{0}^{\infty}\left\{\tilde{E}\left(u, \beta_{0}\right)-\tilde{E}(u, \hat{\beta})\right\} S^{(0)}\left(u, \beta_{0}\right) d \Lambda_{0}(u) .
\end{gathered}
$$

Applying the Taylor formula to the function $\beta \longrightarrow \tilde{E}(u, \beta)$ give

$$
\tilde{E}(u, \hat{\beta})-\tilde{E}\left(u, \beta_{0}\right)=\frac{\partial \tilde{E}\left(u, \beta_{0}\right)}{\partial \beta}\left(\hat{\beta}-\beta_{0}\right) .
$$

That imply

$$
\begin{gathered}
n^{-1 / 2} \hat{U}_{j}=n^{-1 / 2} \sum_{i=1}^{n} \int_{0}^{\infty}\left\{\hat{w}^{(i)}(u)-\tilde{E}\left(u, \beta_{0}\right)\right\} d M_{i}(u)- \\
n^{-1} \int_{0}^{\infty} \frac{\partial \tilde{E}\left(u, \beta_{0}\right)}{\partial \beta} S^{(0)}\left(u, \beta_{0}\right) d \Lambda_{0}(u) n^{1 / 2}\left(\hat{\beta}-\beta_{0}\right)+o_{p}(1)= \\
n^{-1 / 2} \sum_{i=1}^{n} \int_{0}^{\infty}\left\{\hat{w}^{(i)}(u)-\tilde{E}\left(u, \beta_{0}\right)\right\} d M_{i}(u)- \\
\Sigma^{*} \Sigma^{-1} n^{-1 / 2} \sum_{i=1}^{n} \int_{0}^{\infty}\left\{x^{(i)}(u)-E\left(u, \beta_{0}\right)\right\} d M_{i}(u)+o_{p}(1),
\end{gathered}
$$


where $\Sigma$ and $\Sigma^{*}$ are the limits in probability of the random matrices,

$$
\begin{gathered}
\hat{\Sigma}=n^{-1} \int_{0}^{\infty} V(u, \hat{\beta}) d N(u), \quad \hat{\Sigma}^{*}=n^{-1} \int_{0}^{\infty} \tilde{V}(u, \hat{\beta}) d N(u), \\
V(t, \beta)=\frac{S^{(2)}(t, \beta)}{S^{(0)}(t, \beta)}-(E(t, \beta))^{\otimes 2}, \quad \tilde{V}(t, \beta)=\frac{\tilde{S}^{(2)}(t, \beta)}{S^{(0)}(t, \beta)}-\tilde{E}(t, \beta) E^{T}(t, \beta) .
\end{gathered}
$$

So

$$
\begin{gathered}
<n^{-1 / 2} \hat{U}_{j}>=n^{-1} \sum_{i=1}^{n} \int_{0}^{\infty}\left\{\hat{w}^{(i)}(u)-\tilde{E}\left(u, \beta_{0}\right)\right\}^{\otimes 2} e^{\beta_{0}^{T} x^{(i)}(u)} Y_{i}(u) d \Lambda_{0}(u)- \\
2 \Sigma^{*} \Sigma^{-1} n^{-1} \sum_{i=1}^{n} \int_{0}^{\infty}\left\{\hat{w}^{(i)}(u)-\tilde{E}\left(u, \beta_{0}\right)\right\}\left\{x^{(i)}(u)-E\left(u, \beta_{0}\right)\right\}^{T} e^{\beta_{0}^{T} x^{(i)(u)}} \times \\
Y_{i}(u) d \Lambda_{0}(u)+\Sigma^{*} \Sigma^{-1} n^{-1} \sum_{i=1}^{n} \int_{0}^{\infty}\left\{x^{(i)}(u)-E\left(u, \beta_{0}\right)\right\}^{\otimes 2} e^{\beta_{0}^{T} x^{(i)}(u)} \times \\
Y_{i}(u) d \Lambda_{0}(u) \Sigma^{-1}\left(\Sigma^{*}\right)^{T}+o_{p}(1)=\Sigma^{* *}-\Sigma^{*} \Sigma^{-1}\left(\Sigma^{*}\right)^{T}+o_{p}(1),
\end{gathered}
$$

where $\Sigma^{* *}$ is the limit in probability of

$$
\hat{\Sigma}^{* *}=n^{-1} \int_{0}^{\infty} \tilde{\tilde{V}}(u, \hat{\beta}) d N(u)
$$

with

$$
\tilde{\tilde{V}}(u, \beta)=\frac{\tilde{\tilde{S}}^{(2)}(t, \beta)}{S^{(0)}(t, \beta)}-(\tilde{E}(t, \beta))^{\otimes 2} .
$$

Similarly, the Lindeberg condition (see Andersen et al. (1993))

$$
n^{-1} \sum_{i=1}^{n} \int_{0}^{\infty}\left\{\hat{w}_{j}^{(i)}(u)-\tilde{E}_{j}\left(u, \beta_{0}\right)\right\}^{2} \mathbf{1}_{\left\{\mid \hat{w}_{j}^{(i)}(u)-\tilde{E}_{j}\left(u, \beta_{0} \mid \geq \sqrt{n} \varepsilon\right\}\right.} e^{\beta_{0}^{T} x^{(i)}} Y_{i}(u) d \Lambda_{0}(u) \stackrel{P}{\rightarrow} 0 .
$$

is verified. It follows that the stochastic process $n^{-1 / 2} \hat{U}_{j}$ converges in distribution to the zero-mean Gaussian. In particular,

$$
n^{-1 / 2} \hat{U}_{j} \stackrel{\mathcal{D}}{\rightarrow} N(0, D)
$$

where $D=\Sigma^{* *}-\Sigma^{*} \Sigma^{-1}\left(\Sigma^{*}\right)^{T}$.

Finally, the score statistic for testing $H_{0}: \gamma=0$ is

$$
T=\hat{U}_{j}^{2} / \hat{D}
$$

where $\hat{D}=n\left(\hat{\Sigma}^{* *}-\hat{\Sigma}^{*} \hat{\Sigma}^{-1}\left(\hat{\Sigma}^{*}\right)^{T}\right) . T$ is asymptotically $\chi_{1}^{2}$-distributed as $n$ tend to $\infty$. So $H_{0}$ will be rejected with a significance level $\alpha$ if $T>\chi_{1-\alpha}^{2}(1)$ where $\chi_{1-\alpha}^{2}(1)$ is the $(1-\alpha)$ critical value of the $\chi_{1}^{2}$ distribution.

\section{Simulation study}

The primary objective of the small simulation study performed in this section is to investigate the finite sample properties of the suggested test according to different form of added component to Cox model. Specially the level and power properties of the proposed test presented in Section 2 and finalized in section 3, are studied and compared simultaneously with those of the other tests cited in section 1.

In this study, we consider five cases: the first to examine the level properties of considered tests, the second and the third to study the power and the level properties when the ratio of hazard rates is respectively monotonic and nonmonotonic in function of time, the fourth and last case to show the power comportement when violating the proportionality by including a non linear form of covariate component. 
Rejection probabilities are estimated by Monte Carlo simulations, in all cases studied. 5000 repetitions in each case are used with a fixed level for the test is 5\%. This implies that the standard deviation of the reported estimated rejection probabilities is at most $\sqrt{\frac{0.5 \times 0.5}{5000}} \simeq 0.0071$ and for level simulations typically around $\sqrt{\frac{0.05 \times 0.95}{5000}} \simeq 0.0031$.

The simulations are performed in R language (Lafaye de Micheaux et al. (2011)). The both complete and censoring cases are considered. The number of units is $n=50,100$, and 200. The failure time is genereted from the specific model in each case. The used expression to do it is $T_{i}=F_{x_{i}}^{-1}\left(U_{i}\right)$ for any $i=1, \ldots, n$, where $U_{i}$ is the $i$ th element in $n$ generated observations from Uniform[0,1] distribution and $F_{x_{i}}$ is the distribution function of the $i$ th unit.

The choosing probability of censoring is $p=0$ (for complete data), $p=0.2$ and $p=0.5$ (there are approximately $20 \%$ and $50 \%$ censoring units respectively). The censoring time is taken constant of each subject, that means that $C_{i}=d_{i}$, for any $i=1, \ldots, n$. The constant $d_{i}$ verify $S_{x_{i}}\left(d_{i}\right)=p(p \neq 0)$ where $S_{x_{i}}$ is the survival function of the $i$ th unit.

For the $(G)$ test, we use the function cox.zph from the package survival in $\mathrm{R}$ with the default transform argument value which is "km" (the Kaplan-Meier estimate computed from the data without covariates). Among the many choice of the smoothly functions in $(K R)$ test, we use $d$ orthonormal Legendre polynomials provided by orthopolynom package in $\mathrm{R}$ with $d=2,3$, and 4 .

\subsection{Case 1}

We start the study of level properties of the different tests considered by generating failure time $T_{i}(i=1, \ldots, n)$ from the model

$$
\lambda_{x}(t)=2 e^{2 x_{1}+x_{2}},
$$

where the covariates $x_{1}$ and $x_{2}$ are Uniform[0,1] distributed.

The rejection probabilities for different sample sizes $n$ are reported in Table 1.

Table 1: Estimated rejection probabilities for different values of censoring probability $p$ and different sample sizes $n$.

\begin{tabular}{|c|c|c|c|c|c|c|c|c|c|c|c|}
\hline \multirow[b]{3}{*}{$n$} & \multirow[b]{3}{*}{$p$} & \multirow[b]{3}{*}{ Cov. } & \multicolumn{9}{|c|}{ Test } \\
\hline & & & \multirow{2}{*}{$\begin{array}{c}\text { Proposed } \\
\text { test }\end{array}$} & \multirow[t]{2}{*}{$(A D)$} & \multirow[t]{2}{*}{$(C V)$} & \multirow[t]{2}{*}{$(K S)$} & \multirow[t]{2}{*}{$(G)$} & \multirow[t]{2}{*}{$(B A G)$} & \multicolumn{3}{|c|}{$(K R)$} \\
\hline & & & & & & & & & $d=2$ & $d=3$ & $d=4$ \\
\hline \multirow[t]{6}{*}{50} & 0 & $x_{1}$ & 0.0506 & 0.0674 & 0.0620 & 0.0462 & 0.0318 & 0.0568 & 0.0432 & 0.0372 & 0.0320 \\
\hline & & $x_{2}$ & 0.0570 & 0.0618 & 0.0544 & 0.0418 & 0.0370 & 0.0564 & 0.0343 & 0.0268 & 0.0264 \\
\hline & 0.2 & $x_{1}$ & 0.0528 & 0.0532 & 0.0566 & 0.0404 & 0.0246 & 0.0552 & 0.0474 & 0.0410 & 0.0328 \\
\hline & & $x_{2}$ & 0.0550 & 0.0618 & 0.0610 & 0.0444 & 0.0412 & 0.0546 & 0.0398 & 0.0352 & 0.0304 \\
\hline & 0.5 & $x_{1}$ & 0.0566 & 0.0536 & 0.0570 & 0.0372 & 0.0164 & 0.0566 & 0.0480 & 0.0402 & 0.0318 \\
\hline & & $x_{2}$ & 0.0578 & 0.0584 & 0.0586 & 0.0342 & 0.0338 & 0.0534 & 0.0420 & 0.0350 & 0.0262 \\
\hline \multirow[t]{6}{*}{100} & 0 & $x_{1}$ & 0.0532 & 0.0568 & 0.0524 & 0.0442 & 0.0276 & 0.0490 & 0.0378 & 0.0380 & 0.0410 \\
\hline & & $x_{2}$ & 0.0566 & 0.0602 & 0.0568 & 0.0450 & 0.0446 & 0.0564 & 0.0378 & 0.0352 & 0.0340 \\
\hline & 0.2 & $x_{1}$ & 0.0582 & 0.0608 & 0.0602 & 0.0494 & 0.0224 & 0.0526 & 0.0478 & 0.0428 & 0.0416 \\
\hline & & $x_{2}$ & 0.0624 & 0.0646 & 0.0604 & 0.0470 & 0.0458 & 0.0598 & 0.0482 & 0.0398 & 0.0346 \\
\hline & 0.5 & $x_{1}$ & 0.0488 & 0.0486 & 0.0492 & 0.0386 & 0.0112 & 0.0514 & 0.0460 & 0.039 & 0.0346 \\
\hline & & $x_{2}$ & 0.0496 & 0.0596 & 0.0552 & 0.0420 & 0.0318 & 0.0544 & 0.0418 & 0.0350 & 0.0286 \\
\hline \multirow[t]{6}{*}{200} & 0 & $x_{1}$ & 0.0572 & 0.0586 & 0.0576 & 0.0482 & 0.0356 & 0.0574 & 0.0424 & 0.0408 & 0.0418 \\
\hline & & $x_{2}$ & 0.0534 & 0.0570 & 0.0536 & 0.0460 & 0.0444 & 0.0540 & 0.0430 & 0.0362 & 0.0360 \\
\hline & 0.2 & $x_{1}$ & 0.0490 & 0.0476 & 0.0474 & 0.0420 & 0.0174 & 0.0490 & 0.0434 & 0.0402 & 0.0368 \\
\hline & & $x_{2}$ & 0.0498 & 0.0528 & 0.0504 & 0.0474 & 0.0352 & 0.0488 & 0.0440 & 0.0402 & 0.0364 \\
\hline & 0.5 & $x_{1}$ & 0.0508 & 0.0478 & 0.0496 & 0.0364 & 0.0308 & 0.0450 & 0.0448 & 0.0408 & 0.0338 \\
\hline & & $x_{2}$ & 0.0512 & 0.0522 & 0.0534 & 0.0430 & 0.0308 & 0.0450 & 0.0448 & 0.0408 & 0.0338 \\
\hline
\end{tabular}

Table 1 illustrate level properties of the considered tests. We see that the all tests have generally a very good level 
properties even for fairly small sample sizes. The tests $(G),(K S)$ and $(K R)$ are clearly conservative. Specially $(K R)$ becomes more conservative when the number of the used smoothly functions increases which is natural because when including a redundant component that will increases the test critical value with non significative change in the test statistic value.

\section{2. $\operatorname{Cas} 2$}

In this second case we shall examine the level and the power of the test in the same model containing both proportional and non-proportional covariates. The suggested model is

$$
\lambda_{x}(t)=2 e^{(6 t-1) x_{1}+2 x_{2}},
$$

where $x_{1}$ and $x_{2}$ are Uniform[0,2] distributed. It is clear that the proportionality is violated for $x_{1}$ which is due to the added component $6 t x_{1}$. Note that under this model the ratio of hazard rates is monotonic in function of time.

The table 2 contain the probability of rejection of proportionality assumption of $x_{1}$ and $x_{2}$.

Table 2: Estimated rejection probabilities for different values of censoring probability $p$ and different sample sizes $n$.

\begin{tabular}{|c|c|c|c|c|c|c|c|c|c|c|c|}
\hline \multirow[b]{3}{*}{$n$} & \multirow[b]{3}{*}{$p$} & \multirow[b]{3}{*}{ Cov. } & \multicolumn{9}{|c|}{ Test } \\
\hline & & & \multirow{2}{*}{$\begin{array}{c}\text { Proposed } \\
\text { test }\end{array}$} & \multirow[t]{2}{*}{$(A D)$} & \multirow[t]{2}{*}{$(C V)$} & \multirow[t]{2}{*}{$(K S)$} & \multirow[t]{2}{*}{$(G)$} & \multirow[t]{2}{*}{$(B A G)$} & \multicolumn{3}{|c|}{$(K R)$} \\
\hline & & & & & & & & & $d=2$ & $d=3$ & $d=4$ \\
\hline \multirow[t]{6}{*}{50} & 0 & $x_{1}$ & 0.4818 & 0.5602 & 0.5220 & 0.4292 & 0.4768 & 0.5856 & 0.3722 & 0.2816 & 0.2194 \\
\hline & & $x_{2}$ & 0.0512 & 0.0778 & 0.0680 & 0.0490 & 0.0352 & 0.0760 & 0.0468 & 0.0386 & 0.0412 \\
\hline & 0.2 & $x_{1}$ & 0.3022 & 0.3896 & 0.3618 & 0.2802 & 0.3314 & 0.3962 & 0.2210 & 0.1632 & 0.1238 \\
\hline & & $x_{2}$ & 0.0518 & 0.0608 & 0.0564 & 0.0364 & 0.0216 & 0.0670 & 0.0510 & 0.0436 & 0.0384 \\
\hline & 0.5 & $x_{1}$ & 0.1266 & 0.1768 & 0.1700 & 0.1206 & 0.1512 & 0.1708 & 0.0924 & 0.0618 & 0.0474 \\
\hline & & $x_{2}$ & 0.0534 & 0.0676 & 0.0652 & 0.0426 & 0.0164 & 0.0670 & 0.0518 & 0.0412 & 0.0342 \\
\hline \multirow[t]{6}{*}{100} & 0 & $x_{1}$ & 0.8128 & 0.8832 & 0.8608 & 0.7996 & 0.8376 & 0.9034 & 0.7974 & 0.7114 & 0.6270 \\
\hline & & $x_{2}$ & 0.0486 & 0.0776 & 0.0704 & 0.0530 & 0.0428 & 0.0836 & 0.0574 & 0.0518 & 0.0500 \\
\hline & 0.2 & $x_{1}$ & 0.5674 & 0.6866 & 0.6564 & 0.5638 & 0.6476 & 0.7072 & 0.5298 & 0.4304 & 0.3642 \\
\hline & & $x_{2}$ & 0.0462 & 0.0594 & 0.0582 & 0.0454 & 0.0316 & 0.0542 & 0.0566 & 0.0534 & 0.0458 \\
\hline & 0.5 & $x_{1}$ & 0.1720 & 0.3144 & 0.2992 & 0.2318 & 0.1778 & 0.3040 & 0.1828 & 0.1404 & 0.1094 \\
\hline & & $x_{2}$ & 0.0548 & 0.0712 & 0.0682 & 0.0454 & 0.0158 & 0.0804 & 0.0522 & 0.0450 & 0.0350 \\
\hline \multirow[t]{6}{*}{200} & 0 & $x_{1}$ & 0.9826 & 0.9952 & 0.9936 & 0.9858 & 0.9918 & 0.9970 & 0.9890 & 0.9788 & 0.9658 \\
\hline & & $x_{2}$ & 0.0418 & 0.0724 & 0.0702 & 0.0600 & 0.0664 & 0.0772 & 0.0808 & 0.0666 & 0.0700 \\
\hline & 0.2 & $x_{1}$ & 0.8464 & 0.9306 & 0.9160 & 0.8718 & 0.9196 & 0.9416 & 0.8804 & 0.8242 & 0.7692 \\
\hline & & $x_{2}$ & 0.0500 & 0.0660 & 0.0648 & 0.0508 & 0.0508 & 0.0592 & 0.0696 & 0.0716 & 0.0614 \\
\hline & 0.5 & $x_{1}$ & 0.3450 & 0.5444 & 0.5192 & 0.4484 & 0.5110 & 0.5584 & 0.4046 & 0.3246 & 0.2646 \\
\hline & & $x_{2}$ & 0.0444 & 0.0766 & 0.0628 & 0.0532 & 0.0188 & 0.0898 & 0.0632 & 0.0516 & 0.0456 \\
\hline
\end{tabular}

For detecting the non-proportionality of $x_{1}$, all considered tests have not a great difference power except when $n$ take a small value, the (KR) test has a lowest power. In testing the proportionality of $x_{2}$, the $(G)$ test is conservative and it is more conservative if $p$ increases, while the probability of the proportionality rejection of the two tests $(A D)$ and $(C V)$ exceed the fixed level test.

We find also and clearly that the proposed and $(K S)$ tests on the one hand, and $(A D)$ and $(C V)$ on the other hand, have the same results in all cases of $n$ and $p$, and in $(K R)$ test, the power and level decrease when the number of smoothly functions increase.

In conclusion, by comparing and analyzing the different results in table 2, of testing the proportionality of the two covariates $x_{1}$ and $x_{2}$, the proposed and $(K S)$ tests are the best in this case. 


\section{3. $\quad$ Cas 3}

This third case is an example of nonmonotonic ratio of hazard rates in time. The associated model is

$$
\lambda_{x}(t)=e^{0.1 x_{1}-0.75|t-1| x_{1}-0.5 x_{2}},
$$

where $x_{1}$ and $x_{2}$ are Uniform[0,1] distributed. Note that the adding component here is $-0.75|t-1| x_{1}$ which is linear according to $x_{1}$. The table 3 show the results of testing the proportionality assumption of each one of the covariates $x_{1}$ and $x_{2}$.

Table 3: Estimated rejection probabilities for different values of censoring probability $p$ and different sample sizes $n$.

\begin{tabular}{|c|c|c|c|c|c|c|c|c|c|c|c|}
\hline \multirow[b]{3}{*}{$n$} & \multirow[b]{3}{*}{$p$} & \multirow[b]{3}{*}{ Cov. } & \multicolumn{9}{|c|}{ Test } \\
\hline & & & \multirow{2}{*}{$\begin{array}{c}\text { Proposed } \\
\text { test }\end{array}$} & \multirow[t]{2}{*}{$(A D)$} & \multirow[t]{2}{*}{$(C V)$} & \multirow[t]{2}{*}{$(K S)$} & \multirow[t]{2}{*}{$(G)$} & \multirow[t]{2}{*}{$(B A G)$} & \multicolumn{3}{|c|}{$(K R)$} \\
\hline & & & & & & & & & $d=2$ & $d=3$ & $d=4$ \\
\hline \multirow[t]{6}{*}{50} & 0 & $x_{1}$ & 0.3716 & 0.4276 & 0.3598 & 0.2496 & 0.3244 & 0.3930 & 0.2926 & 0.2486 & 0.1952 \\
\hline & & $x_{2}$ & 0.0598 & 0.0654 & 0.0596 & 0.0424 & 0.0424 & 0.0558 & 0.0498 & 0.0396 & 0.0346 \\
\hline & 0.2 & $x_{1}$ & 0.2648 & 0.3132 & 0.2820 & 0.1958 & 0.2694 & 0.3070 & 0.2854 & 0.2190 & 0.1620 \\
\hline & & $x_{2}$ & 0.0488 & 0.0506 & 0.0494 & 0.0380 & 0.0386 & 0.0468 & 0.0532 & 0.0508 & 0.0438 \\
\hline & 0.5 & $x_{1}$ & 0.2546 & 0.2926 & 0.2966 & 0.2010 & 0.2600 & 0.2978 & 0.3134 & 0.2056 & 0.1624 \\
\hline & & $x_{2}$ & 0.0546 & 0.0436 & 0.0450 & 0.026 & 0.0326 & 0.0460 & 0.0558 & 0.0450 & 0.0356 \\
\hline \multirow[t]{6}{*}{100} & 0 & $x_{1}$ & 0.6754 & 0.7596 & 0.6514 & 0.5228 & 0.6416 & 0.6932 & 0.6342 & 0.7182 & 0.6484 \\
\hline & & $x_{2}$ & 0.0532 & 0.0528 & 0.0498 & 0.0464 & 0.0410 & 0.0504 & 0.0490 & 0.0490 & 0.0422 \\
\hline & 0.2 & $x_{1}$ & 0.4872 & 0.6160 & 0.5070 & 0.4142 & 0.4858 & 0.5374 & 0.6126 & 0.6586 & 0.5445 \\
\hline & & $x_{2}$ & 0.0510 & 0.0536 & 0.0550 & 0.0412 & 0.0462 & 0.0562 & 0.0518 & 0.0454 & 0.0404 \\
\hline & 0.5 & $x_{1}$ & 0.5198 & 0.6348 & 0.5870 & 0.4926 & 0.5112 & 0.5646 & 0.7146 & 0.6442 & 0.5472 \\
\hline & & $x_{2}$ & 0.0552 & 0.0520 & 0.0530 & 0.0374 & 0.0388 & 0.0540 & 0.0538 & 0.0450 & 0.0392 \\
\hline \multirow[t]{6}{*}{200} & 0 & $x_{1}$ & 0.9408 & 0.9800 & 0.9272 & 0.8772 & 0.9262 & 0.9446 & 0.9358 & 0.9870 & 0.9878 \\
\hline & & $x_{2}$ & 0.0548 & 0.0544 & 0.0522 & 0.0398 & 0.0458 & 0.0542 & 0.0490 & 0.0492 & 0.0464 \\
\hline & 0.2 & $x_{1}$ & 0.8012 & 0.9354 & 0.8354 & 0.8090 & 0.7926 & 0.8360 & 0.9290 & 0.9748 & 0.9748 \\
\hline & & $x_{2}$ & 0.0458 & 0.0450 & 0.0474 & 0.0426 & 0.0378 & 0.0450 & 0.0514 & 0.0542 & 0.0510 \\
\hline & 0.5 & $x_{1}$ & 0.8400 & 0.9498 & 0.9022 & 0.8746 & 0.8116 & 0.8498 & 0.9730 & 0.9830 & 0.9696 \\
\hline & & $x_{2}$ & 0.0514 & 0.0544 & 0.0564 & 0.0438 & 0.0400 & 0.0582 & 0.0512 & 0.0480 & 0.0448 \\
\hline
\end{tabular}

We see in Table 3 that all considered tests has a similar and large power for detecting a nonmonotonic deviation of the proportionality assumption, and they give a probability of rejection near to the fixed level in testing the proportionality of the proportional covariate $x_{2}$.

\subsection{Cas 4}

As mentionned in section 2, the violation of the proportionality can be caused not only by time dependent coefficients as in case 1-3, but may be by a non-linear form of the added component in Cox model. Our aim here is to study the power comportement of the tests cited in this article according to a nonlinear added component in function of $x$. For that we propose the following simple model with one covariate:

$$
\lambda_{x}(t)=e^{0.5 x+x^{a} t},
$$

where $x$ is Uniform[0,2] distributed and $a$ present the non-linearity degree taking the following values: $0,1,2,3,4$, 5. In this case $n$ is fixed at 100, and $p$ take the two values: 0 and 0.2. The results of the performed simulations are resumed in the table 4 . 
Table 4: Estimated rejection probabilities for different values of both probability of censoring $p$ and $a$.

\begin{tabular}{|c|c|c|c|c|c|c|c|c|c|c|}
\hline \multirow[b]{3}{*}{$p$} & \multirow[b]{3}{*}{$a$} & & \multicolumn{8}{|c|}{ Test } \\
\hline & & \multirow{2}{*}{$\begin{array}{c}\text { Proposed } \\
\text { test }\end{array}$} & \multirow[t]{2}{*}{$(A D)$} & \multirow[t]{2}{*}{$(C V)$} & \multirow[t]{2}{*}{$(K S)$} & \multirow[t]{2}{*}{$(G)$} & \multirow{2}{*}{$(B A G)$} & \multicolumn{3}{|c|}{$(K R)$} \\
\hline & & & & & & & & $d=2$ & $d=3$ & $d=4$ \\
\hline \multirow[t]{6}{*}{0} & 0 & 0.0520 & 0.0518 & 0.0496 & 0.0404 & 0.0378 & 0.0532 & 0.0430 & 0.0392 & 0.0374 \\
\hline & 1 & 0.2268 & 0.2874 & 0.2632 & 0.1952 & 0.1964 & 0.2996 & 0.1758 & 0.1250 & 0.0998 \\
\hline & 2 & 0.4746 & 0.4126 & 0.4032 & 0.3252 & 0.2814 & 0.3630 & 0.2180 & 0.1636 & 0.1290 \\
\hline & 3 & 0.7006 & 0.4110 & 0.4130 & 0.3660 & 0.2552 & 0.2430 & 0.1850 & 0.1668 & 0.1296 \\
\hline & 4 & 0.8944 & 0.3422 & 0.3252 & 0.3156 & 0.1592 & 0.0996 & 0.1030 & 0.1254 & 0.1168 \\
\hline & 5 & 0.9594 & 0.2682 & 0.2150 & 0.2450 & 0.0662 & 0.0662 & 0.0420 & 0.0742 & 0.0812 \\
\hline \multirow[t]{6}{*}{0.2} & 0 & 0.0540 & 0.0524 & 0.0558 & 0.0410 & 0.0290 & 0.0576 & 0.0470 & 0.0418 & 0.0374 \\
\hline & 1 & 0.1050 & 0.1080 & 0.111 & 0.0834 & 0.0544 & 0.1136 & 0.0846 & 0.0628 & 0.0536 \\
\hline & 2 & 0.1946 & 0.1704 & 0.1680 & 0.1330 & 0.0768 & 0.1462 & 0.1060 & 0.0840 & 0.0678 \\
\hline & 3 & 0.3460 & 0.1846 & 0.1926 & 0.1616 & 0.0798 & 0.1092 & 0.1136 & 0.0958 & 0.0802 \\
\hline & 4 & 0.5202 & 0.1902 & 0.1806 & 0.1724 & 0.0568 & 0.0660 & 0.0974 & 0.1026 & 0.0928 \\
\hline & 5 & 0.6704 & 0.1886 & 0.1534 & 0.1696 & 0.0442 & 0.0892 & 0.0768 & 0.0920 & 0.0898 \\
\hline
\end{tabular}

As shown in Table 4, for $a=0$ all the tests give almost the same probability of rejection which is close to the fixed level. This is natural since $a=0$ corresponds to the proportionality hypothesis and the corresponding results are already discussed in case 1 .

For $a=1$, all the tests almost have similar power except that of the $(K R)$ test with $d=3$ and $d=4$.

When a exceeds 1 , the proposed test has shown that it is the powerfull compared to other tests and the difference between its power and that of other tests becomes clearer when $a$ increases.

\subsection{Cas 5}

This case was included in order to confirm the results obtained in case 4 by considering two covariates one is nonproportional caused by adding a non-linear component according to herself and the other is proportional. The proposed model is

$$
\lambda_{x}(t)=e^{0.5 x_{1}+\frac{4}{x_{1}} t+0.5 x_{2}},
$$

where $x_{1}$ and $x_{2}$ are Uniform[0,1] distributed.

The results of the performed simulations are reported in the table 5 .

The obtained results in this case show the great power of the proposed test based on alternative model (7) to distinguish between the proportional and non proportional covariates in (9). The $(A D),(C V),(K S)$ and $(B A G)$ is not able to have this properties with their very poor power as shown in table 5 while the $(G)$ and $(K R)$ tests asserted the proportionality in the two covariates which is shown in their rejection probabilities close to the fixed level for the different values of $n$ and $p$.

\subsection{Comments}

The simulation study carried out in this article clearly showed in testing the proportionality of specified covariate that:

First, the proposed test give the same results as the existing tests, if this covariate is proportional (its corresponding coefficient is constant in time) or not (its coefficient is time dependent, which means, introduction into the cox model, of a time and linear component with respect to the covariate concerned by the test).

Second, the proposed test proved that it is the only one among all the tests cited here able of detecting the nonproportionality of a covariate which due to the existence, in the cox model, of a time and non-linear component with respect to this covariate. 
Table 5: Estimated rejection probabilities for different values of censoring probability $p$ and different sample sizes $n$.

\begin{tabular}{|c|c|c|c|c|c|c|c|c|c|c|c|}
\hline \multirow[b]{3}{*}{$n$} & \multirow[b]{3}{*}{$p$} & \multirow[b]{3}{*}{ Cov. } & \multicolumn{9}{|c|}{ Test } \\
\hline & & & \multirow{2}{*}{$\begin{array}{c}\text { Proposed } \\
\text { test }\end{array}$} & \multirow[t]{2}{*}{$(A D)$} & \multirow[t]{2}{*}{$(C V)$} & \multirow[t]{2}{*}{$(K S)$} & \multirow[t]{2}{*}{$(G)$} & \multirow[t]{2}{*}{$(B A G)$} & \multicolumn{3}{|c|}{$(K R)$} \\
\hline & & & & & & & & & $d=2$ & $d=3$ & $d=4$ \\
\hline \multirow[t]{6}{*}{50} & 0 & $x_{1}$ & 0.4250 & 0.1012 & 0.0976 & 0.1018 & 0.0526 & 0.097 & 0.0512 & 0.0468 & 0.0476 \\
\hline & & $x_{2}$ & 0.0548 & 0.0582 & 0.0500 & 0.0372 & 0.0368 & 0.0520 & 0.0416 & 0.0388 & 0.0348 \\
\hline & 0.2 & $x_{1}$ & 0.3796 & 0.1116 & 0.1082 & 0.1028 & 0.0632 & 0.0998 & 0.0562 & 0.0532 & 0.0520 \\
\hline & & $x_{2}$ & 0.0566 & 0.0512 & 0.0500 & 0.0360 & 0.0398 & 0.0492 & 0.0506 & 0.0448 & 0.0372 \\
\hline & 0.5 & $x_{1}$ & 0.2172 & 0.0882 & 0.0906 & 0.0770 & 0.0540 & 0.0786 & 0.0566 & 0.0468 & 0.0408 \\
\hline & & $x_{2}$ & 0.0534 & 0.0474 & 0.0484 & 0.0344 & 0.0404 & 0.0494 & 0.0522 & 0.0424 & 0.0358 \\
\hline \multirow[t]{6}{*}{100} & 0 & $x_{1}$ & 0.6594 & 0.1048 & 0.0970 & 0.1140 & 0.0560 & 0.1000 & 0.0456 & 0.0484 & 0.0454 \\
\hline & & $x_{2}$ & 0.0540 & 0.0560 & 0.0546 & 0.0416 & 0.0428 & 0.0486 & 0.0470 & 0.0424 & 0.0408 \\
\hline & 0.2 & $x_{1}$ & 0.6556 & 0.1140 & 0.1096 & 0.1130 & 0.0602 & 0.0950 & 0.0590 & 0.0582 & 0.0518 \\
\hline & & $x_{2}$ & 0.0542 & 0.0580 & 0.0554 & 0.0428 & 0.0460 & 0.0550 & 0.0470 & 0.0410 & 0.0432 \\
\hline & 0.5 & $x_{1}$ & 0.3946 & 0.1016 & 0.0974 & 0.0960 & 0.0566 & 0.0838 & 0.0580 & 0.0512 & 0.0478 \\
\hline & & $x_{2}$ & 0.0514 & 0.0512 & 0.0476 & 0.0362 & 0.0376 & 0.0502 & 0.0518 & 0.0460 & 0.0398 \\
\hline \multirow[t]{6}{*}{200} & 0 & $x_{1}$ & 0.9056 & 0.1168 & 0.0990 & 0.1274 & 0.0586 & 0.1006 & 0.0490 & 0.0458 & 0.0482 \\
\hline & & $x_{2}$ & 0.0592 & 0.0572 & 0.0546 & 0.0494 & 0.0472 & 0.0504 & 0.0472 & 0.0476 & 0.0414 \\
\hline & 0.2 & $x_{1}$ & 0.9116 & 0.1314 & 0.1144 & 0.1308 & 0.0650 & 0.1000 & 0.0672 & 0.0646 & 0.0646 \\
\hline & & $x_{2}$ & 0.0522 & 0.0532 & 0.0508 & 0.0440 & 0.0430 & 0.0486 & 0.0470 & 0.0498 & 0.0476 \\
\hline & 0.5 & $x_{1}$ & 0.6704 & 0.0998 & 0.0918 & 0.1010 & 0.0528 & 0.0814 & 0.0572 & 0.0564 & 0.0540 \\
\hline & & $x_{2}$ & 0.0512 & 0.0456 & 0.0496 & 0.0418 & 0.0360 & 0.0494 & 0.0514 & 0.0466 & 0.0430 \\
\hline
\end{tabular}

\section{Real data analysis}

For illustrating application on real data of the proposed test compared to the cited tests in this article, we use three examples. To calculate the p-value of $(A D),(C V)$, and $(K S)$ we use respectively the following functions: $\mathrm{pAD}$, pCvM (both are from goftest package), and pkolmim (from kolmim package).

\subsection{Example 1 (Gastric cancer data):}

Stablein and Koutrouvelis (1985) studied the well-known two-sample data of the Gastrointestinal Tumor Study Group concerning effects of chemotherapy $(x=0)$ and chemotherapy plus radiotherapy $(x=1)$ on the survival times of gastric cancer patients. The number of patients is $n=90$. The data are right-censored. The p-values of the tests are reported in the table 6 .

Table 6: P-value of test statistic in testing the proportionality assumption for treatmen covariate in gastric cancer data.

\begin{tabular}{|c|c|c|c|c|c|c|c|c|c|}
\hline \multirow[b]{3}{*}{ Covariable } & \multicolumn{9}{|c|}{ Test } \\
\hline & Proposed & $(A D)$ & $(C V)$ & $(K S)$ & $(G)$ & $(B A G)$ & & $(K R)$ & \\
\hline & test & & & & & & $d=2$ & $d=3$ & $d=4$ \\
\hline treatment & 0.0041 & 0.0004 & 0.0003 & 0.0006 & 0.0003 & 0.0003 & 0.0018 & 0.0051 & 0.0065 \\
\hline
\end{tabular}

The proportionality assumption of the chemo and chemo-radiotherapy is strongly rejected by all tests. The result is natural because the Kaplan-Meier estimators of the survival functions of two patient groups intersect (see for instance Stablein and Koutrouvelis (1985) and Bagdonavičius et al. (2004)). 


\subsection{Example 2 (The PBC data):}

The primary biliary cirrhosis (PBC) data from the Mayo Clinic is a fatal chronic liver disease, and out of the 418 patients followed in the study, 161 died before study closure. A listing of the data is found in survival package in R. In a study of the data by Dickson et al. (1989) a proportional hazards model with the five covariates age, edema, $\log ($ bilirubin), log(protime) and log(albumin) was fitted. By applying the coxph function from survival package in $\mathrm{R}$, the results of fitted covariates are

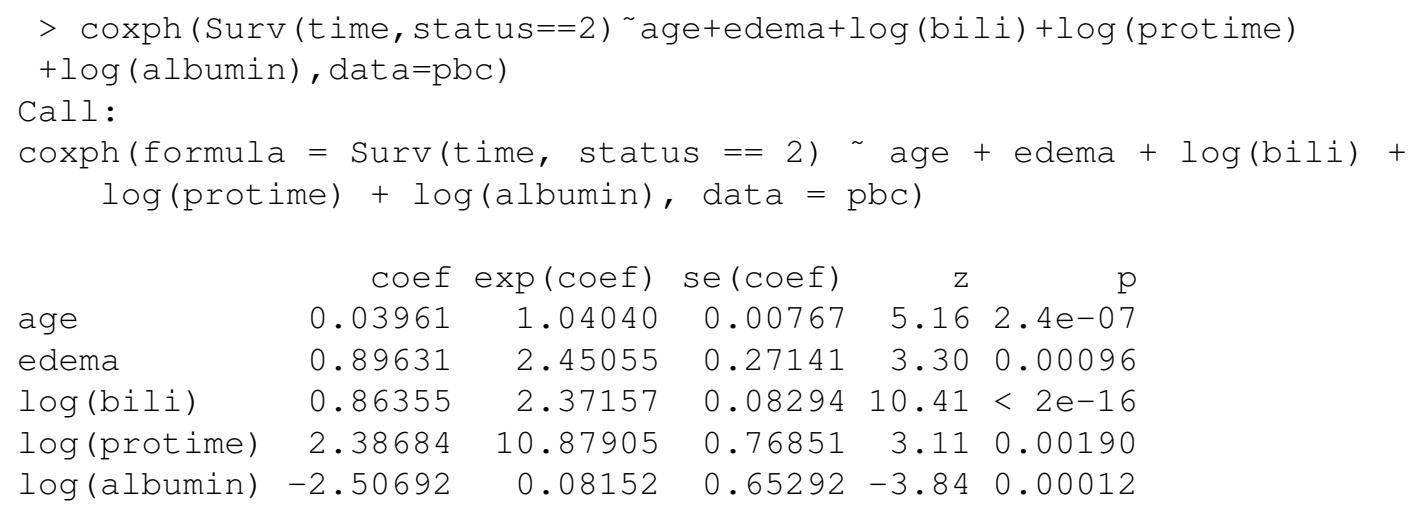

All covariates have a significant effect in Cox model as shown in above fit results. We now examine the proportionality assumption for each covariate among the five covariates. By applying the cited tests, the $p$-value of the corresponding test statistics are reported in the table 7.

Table 7: P-value of test statistic in testing the proportionality assumption for age, edema, log(bili), $\log$ (protime), and $\log (a l b u m i n)$ covariates in PBC data.

\begin{tabular}{|c|c|c|c|c|c|c|c|c|c|}
\hline \multirow[b]{3}{*}{ Covariable } & \multicolumn{9}{|c|}{ Test } \\
\hline & Proposed & $(A D)$ & $(C V)$ & $(K S)$ & $(G)$ & $(B A G)$ & & $(K R)$ & \\
\hline & test & & & & & & $d=2$ & $d=3$ & $d=4$ \\
\hline age & 0.2835 & 0.7334 & 0.6252 & 0.5768 & 0.9622 & 0.8203 & 0.2783 & 0.4430 & 0.4663 \\
\hline edema & 0.0541 & 0.0447 & 0.0356 & 0.0194 & 0.3870 & 0.1625 & 0.0860 & 0.1207 & 0.0088 \\
\hline $\log (b i l i)$ & 0.2045 & 0.2388 & 0.2110 & 0.1489 & 0.1329 & 0.2667 & 0.3775 & 0.4005 & 0.2218 \\
\hline $\log ($ protime) & 0.0005 & 0.0009 & 0.0009 & 0.0043 & 0.0010 & 0.0187 & 0.0004 & 0.0003 & 0.0008 \\
\hline $\log (a \operatorname{lbumin})$ & 0.4325 & 0.4837 & 0.4608 & 0.5380 & 0.6557 & 0.2775 & 0.4960 & 0.2918 & 0.4305 \\
\hline
\end{tabular}

We see that on the one hand all tests strongly reject the proportionality assumption in log (protime) covariate and show its validation for age, $\log (\mathrm{bili})$, and $\log (\mathrm{albumin})$ covariates. On the other hand, we note that only the proposed test and $(A D),(C V),(K S)$ tests can detect non-proportionality in edema while the $(K R)$ test detect the same result if the number of used smoothly functions is well chosen.

\subsection{Example 3 (The Colon cancer data):}

A national intergroup trial was conducted in the 1980's to study the drugs levamisole and fluorouracil for adjuvant therapy of resected colon carcinoma. In the study, 929 patients with stage $\mathrm{C}$ disease were randomly assigned to observation, levamisole alone, or levamisole combined with fluorouracil (treatment covariate). The time to cancer recurrence and the survival time were both considered important outcome measures. There are two rows per person, indicated by the event type (et ype) covariate - et ype $==1$ indicates that row corresponds to recurrence; et ype $==2$ indicates death.

In this trial, 315, 310 and 304 patients received observation, levamisole alone, and levamisole combined with fluorouracil, respectively. The data are imported from survival package and each subjet is described by 10 covariates other than treatment. This data was modeled by the Cox model of mutivariate failure time (recurrence time, death times) in Lin (1994). 
Our aim here is to test the proportionality of each individual covariate in these data. That will be performed on two subsets of original data. The first is the survival time data and the second is the recurrence cancer time data noted respectively colon_death and colon_recurr. To obtain those two subsets, we filter the original data according to the etype covariate. Then we eliminate the patients having a NA value in any of their described covariates in both two subsets. We note the obtained subsets respectively colon_death1 and colon_recurrl. They have 888 patients in each one. In the categorical treatment covariate (noted in colon data rx) we take 1, 2, and 3 values respectively for Obs (observation), Lev (levamisole), and Lev+5FU ( levamisole combined with fluorouracil) categories.

\subsubsection{Survival time data}

In this part, we are interested in the survival time data subset colon_death1. Among the 10th covariates in data we use the backward elimination procedure to select those that are significant in Cox model. The last step in this procedure give the following result:

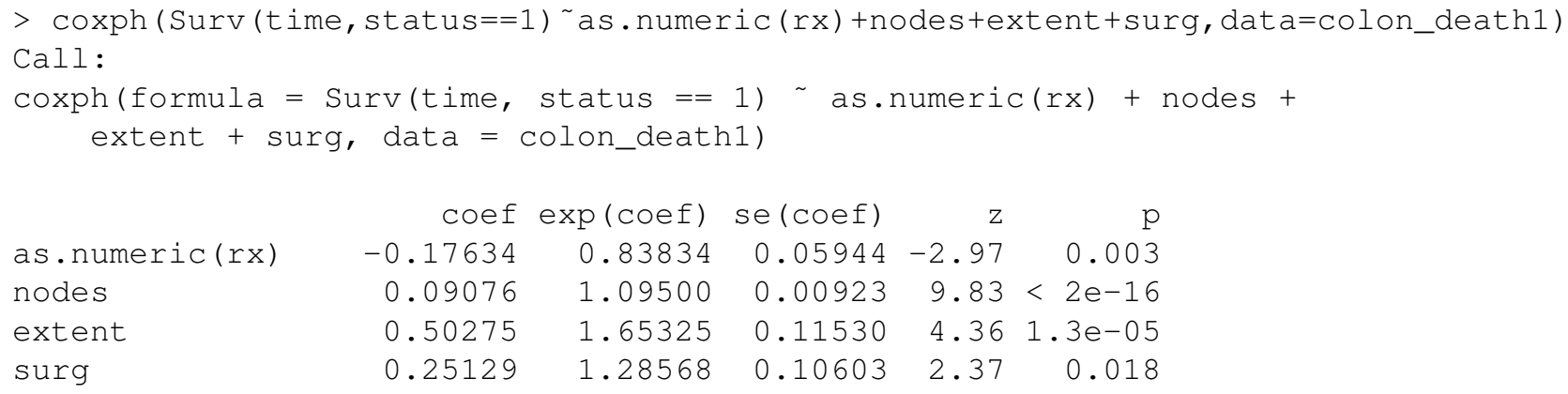

The following table contain the obtained test statistic p-values in testing the proportionality hypothesis by the different tests presented in this article.

Table 8: P-value of test statistic in testing the proportionality assumption for $r x$ (treatment), nodes, extent, and surg covariates in suvival time data

\begin{tabular}{c|cccccccccc}
\hline & \multicolumn{9}{|c}{ Test } \\
\cline { 2 - 7 } Covariable & $\begin{array}{c}\text { Proposed } \\
\text { test }\end{array}$ & $(A D)$ & $(C V)$ & $(K S)$ & $(G)$ & $(B A G)$ & & \multicolumn{3}{c}{$(K R)$} \\
\hline treatment & 0.0551 & 0.1761 & 0.2066 & 0.3746 & 0.1365 & 0.1711 & & 0.2544 & 0.2896 & 0.4297 \\
nodes & 0.0017 & 0.9799 & 0.9833 & 0.9965 & 0.8190 & 0.8812 & & 0.9813 & 0.9888 & 0.3381 \\
extent & 0.0686 & 0.0765 & 0.0658 & 0.1489 & 0.0497 & 0.0564 & & 0.1329 & 0.2481 & 0.3870 \\
surg & 0.5305 & 0.7424 & 0.7978 & 0.8609 & 0.7617 & 0.9664 & & 0.4652 & 0.6721 & 0.7780 \\
\hline
\end{tabular}

We see in table 8 that the proportionality hypothesis is accepted by all tests in surg covariate, also the proposed test and $(A D),(C V),(G)$ and $(B A G)$ tests reject it with a fixed level $10 \%$ in extent covariate while $(K S)$ and $(K R)$ tests accept it. The importante result here is that the proposed test is the only one that detected the violation of proportionality assumption in treatment cavariate (for fixed level equal to 10\%) and strongly in nodes covariate.

\subsubsection{Recurrence time data}

By using the backward elimination procedure in colon_recurrl data we obtained the same significant covariates as in colon_death1. The results of fitted covariates by cox model are

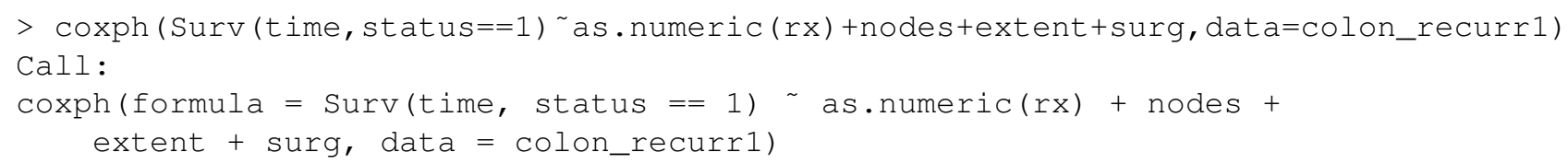




$\begin{array}{lrrrrr} & \text { coef } & \text { exp (coef) } & \text { se (coef) } & \text { z } & p \\ \text { as.numeric (rx) } & -0.23891 & 0.78749 & 0.05849 & -4.08 & 4.4 e-05 \\ \text { nodes } & 0.08130 & 1.08469 & 0.00919 & 8.85 & <2 e-16 \\ \text { extent } & 0.50968 & 1.66476 & 0.11611 & 4.39 & 1.1 e-05 \\ \text { surg } & 0.23260 & 1.26188 & 0.10379 & 2.24 & 0.025\end{array}$

Now we perform the cited tests on colon_recurrl data to test the hypothesis of proportionality of each of the covariates. The p-values of the test statistics are calculated and resumed in the table 9 The results in the table 9 shown

Table 9: P-value of test statistic in testing the proportionality assumption for $r x$ (treatment), nodes, extent, and surg covariates in recurrence time data.

\begin{tabular}{c|cccccccccc}
\hline & \multicolumn{9}{|c}{ Test } \\
\cline { 2 - 7 } Covariable & $\begin{array}{c}\text { Proposed } \\
\text { test }\end{array}$ & $(A D)$ & $(C V)$ & $(K S)$ & $(G)$ & $(B A G)$ & & \multicolumn{3}{c}{$(K R)$} \\
\hline rx & 0.8539 & 0.7460 & 0.7085 & 0.8920 & 0.7190 & 0.8888 & & 0.4773 & 0.6427 & 0.6292 \\
nodes & 0.0004 & 0.3699 & 0.3529 & 0.7384 & 0.3618 & 0.3061 & & 0.1757 & 0.2934 & 0.3633 \\
extent & 0.9157 & 0.3995 & 0.3732 & 0.4218 & 0.7802 & 0.5845 & & 0.1073 & 0.1885 & 0.2743 \\
surg & 0.1252 & 0.2792 & 0.3091 & 0.6179 & 0.1908 & 0.1754 & & 0.4048 & 0.3532 & 0.4368 \\
\hline
\end{tabular}

that all tests accept the proportionality in all covariates, except the proposed test which the only one detecting the non proportionality in nodes.

\section{Conclusion}

To test the proportionality assumption for specified covariate, an alternative model of the Cox model is considered. This alternative is motivated by that the considered tests based on alternatives suppose that the violation of the proportionality assumption is due to an added time component with a linear form according to the covariate concerned by the test while this added component can to have a non linear form. The properties of the proposed alternative model are discussed, the test statistic based on it, is based on score function and it is asymptotically $\chi_{1}^{2}$-distributed. To compare the power of the proposed test against the existing tests, a simulation study is performed using five cases. The obtained results shown that the proposed test is almost powerfull compared to the others tests specially when the added component to the cox model have a non linear form. In the end, three real data are considered. For some covariates the proposed test is the only one that detect the non proportionality in them.

\section{References}

1. Andersen, P., Borgan, O., Gill, R., and Keiding, N. (1993). Statistical Models Based on Counting Processes. Springer, New York.

2. Bagdonavičius, V., Hafdi, M. A., and Nikulin, M. (2004). Analysis of survival data with cross-effects of survival functions. Biostatistics, 5:415-425.

3. Bagdonavičius, V. and Levuliene, R. (2019). Testing proportional hasards for specified covariates. Modern Stochastics: Theory and Applications, 0:1-17.

4. Cox, D. R. (1972). Regression models and life tables. J. R. Statist. Soc., B, 34:187-220.

5. Dickson, E. R., Grambsch, P. M., Fleming, T. R., Fisher, L. D., and Langworthy, A. (1989). Prognosis in primary biliary cirrhosis: Model for decision making. Hepatology, 10:1-7.

6. Grambsch, P. and Therneau, T. M. (1994). Proportional hazards tests and diagnostics based on weighted residuals. Biometrika, 81:515-526.

7. Kraus, D. (2007). Data-driven smooth tests of the proportional hazards assumption. Lifetime Data Anal, $13: 1-16$.

8. Kvaløy, J. T. and Neef, L. R. (2004). Tests for the proportional intensity assumption based on the score process. Lifetime Data Analysis, 10:139-157. 
9. Lafaye de Micheaux, P., Drouilhet, R., and Liquet, B. (2011). Le logiciel R: Maîtriser le language-Effectuer des analyses statistiques. Collection: Statistique et probabilités appliquées, Springer-Verlag, France.

10. Lin, D. Y. (1991). Goodness of fit analysis for the cox regression model based on a class of parameter estimators. J. Am. Stat. Assoc., 86:725-728.

11. Lin, D. Y. (1994). Cox regression analysis of multivariate failure time data: the marginal approach. Statistics in Medicine, 9:2233-22475.

12. Moreau, T., O’Quigley, J., and Mesbah, M. (1985). A global goodness-of-fit statistic for the proportional hazards model. Biometrics, 34:212-218.

13. Nagelkerke, N. J., Oosting, J., and M., H. A. A. (1984). A simple test for goodness of fit of cox's proportional hazards model. Biometrics, 40:483-486.

14. Peña, E. (1998). Smooth goodness-of-fit tests for composite hypothesis in hazard based models. Ann Stat, 26:1935-1971.

15. Quantin, C., Moreau, T., Asselain, B., and Lellouch, J. (1996). A regression model for testing the proportional hazards hypothesis. Biometrics, 52:874-885.

16. Stablein, D. M. and Koutrouvelis, I. A. (1985). A two sample test sensitive to crossing hazards in uncensored and singly censored data. Biometrics, 41:643-652. 